\title{
Quantitative Analysis of Cardiomyocyte Dynamics with Optical Coherence Phase Microscopy
}

\author{
Rehman Ansari*a, ${ }^{\text {ac }}$, Redouane Aherrahrou ${ }^{\mathrm{b}}$, Zouhair Aherrahrou ${ }^{\mathrm{b}}$, Jeanette Erdmann ${ }^{\mathrm{b}}$, Gereon \\ Hüttmann ${ }^{\mathrm{a}}$, and Achim Schweikard ${ }^{\mathrm{C}}$ \\ ${ }^{\mathrm{a} I n s t i t u t ~ f u ̈ r ~ B i o m e d i z i n i s c h e ~ O p t i k, ~}{ }^{\mathrm{b}}$ Medizinische Klinik II Kardiovaskuläre Genetik, and ${ }^{\mathrm{c} I n s t i t u t}$ \\ für Robotik and Kognitive Systeme, Unversity of Lübeck, Lübeck D-23538
}

\begin{abstract}
Spectral domain optical coherence microscopy (OCM) is an interferometric imaging technique for three-dimensional reconstruction of biological samples. Phase sensitive implementation of OCM has generally been in common path interferometer configuration to obtain high phase stability, which limits the numerical aperture of the imaging optics and the transverse resolution. Here, we describe the implementation of optical coherence phase microscope in asymmetric Linnik interferometer configuration, which provides phase stability of 0.5 milliradians along with high spatial resolution. Three-dimensional structural images and dynamic displacement images obtained from spontaneously active cardiomyocytes demonstrate that the phase information could potentially be used for quantitative analysis of contraction dynamics, spatially resolved to sub-cellular structures.
\end{abstract}

Keywords: Optical coherence microscopy, Optical coherence phase microscopy, cardiomyocytes, cellular physiology, cell dynamics

\section{INTRODUCTION}

Spectral domain optical coherence tomography ${ }^{1,2}(\mathrm{OCT})$ is an imaging technique based on low coherence interferometry, where the axial resolution is determined by coherence length of the light source and the transverse resolution is limited by numerical aperture of imaging optics. Spectral domain optical coherence microscopy ${ }^{3,4}$ (OCM) combines OCT with the principles of confocal microscopy, and the compound coherence and confocal gating provides sharp optical sectioning of highly scattering samples such as biological tissue. Optical coherence phase microscopy (OCPM) is a phase sensitive implementation of OCM, where the phase component of the fringe pattern is sensitive to sub-wavelength changes in optical pathlength. In biological tissue, inter and intracellular physiological processes are accompanied with transient changes in optical pathlength. Quantitative measurement of these transient changes in optical pathlength with the phase contrast of OCPM could provide an insight into the cellular physiology. The other advantages of OCPM are that, it is a non-invasive imaging technique and it does not require any extrinsic contrast agents.

\section{EXPERIMENTAL SETUP}

The schematic of the phase sensitive optical coherence phase microscope (OCPM) is as depicted in figure 1. The optical setup is configured in asymmetric Linnik interferometer topology, with a microscope objective in sample arm and dispersion matching substrate and a focusing lens in the reference arm. The low coherence light source is a high power super-luminescent diode (SLD) with center wavelength of $840 \mathrm{~nm}$ and a FWHM bandwidth of $50 \mathrm{~nm}$, yielding an axial resolution of $6.2 \mu \mathrm{m}$ in air. Water immersion microscope objective (Carl Zeiss Microimaging GmbH) with 0.8 NA is used to focus the beam on sample, which provides a transverse spatial resolution of $1 \mu \mathrm{m}$.

*ansari@rob.uni-luebeck.de

Optical Coherence Tomography and Coherence Domain Optical Methods in Biomedicine XVI,

edited by Joseph A. Izatt, James G. Fujimoto, Valery V. Tuchin, Proc. of SPIE Vol. 8213, 821338

(C) 2012 SPIE - CCC code: 1605-7422/12/\$18 - doi: 10.1117/12.911781

Proc. of SPIE Vol. $8213821338-1$ 
X-Y galvanometer scanner unit (Cambridge Technology, U.S.A) is used to steer the focused spot transversely on the sample to obtain en-face optical sections and a linear motor (M-125, Physik Instrumente $\mathrm{GmbH}$ ) is used to translate the sample axially so that stacks of en-face optical sections can be obtained for 3D reconstruction. High phase sensitivity is achieved by splitting the light beam after it passes through the galvanometer scanners. The phase stability of the setup is 0.5 milliradians, corresponding to optical path length sensitivity of $37 \mathrm{pm}$ axially. High speed CMOS line scan camera (Basler sprint, 140k) allows an imaging speed of up to 200k Ascans/sec.

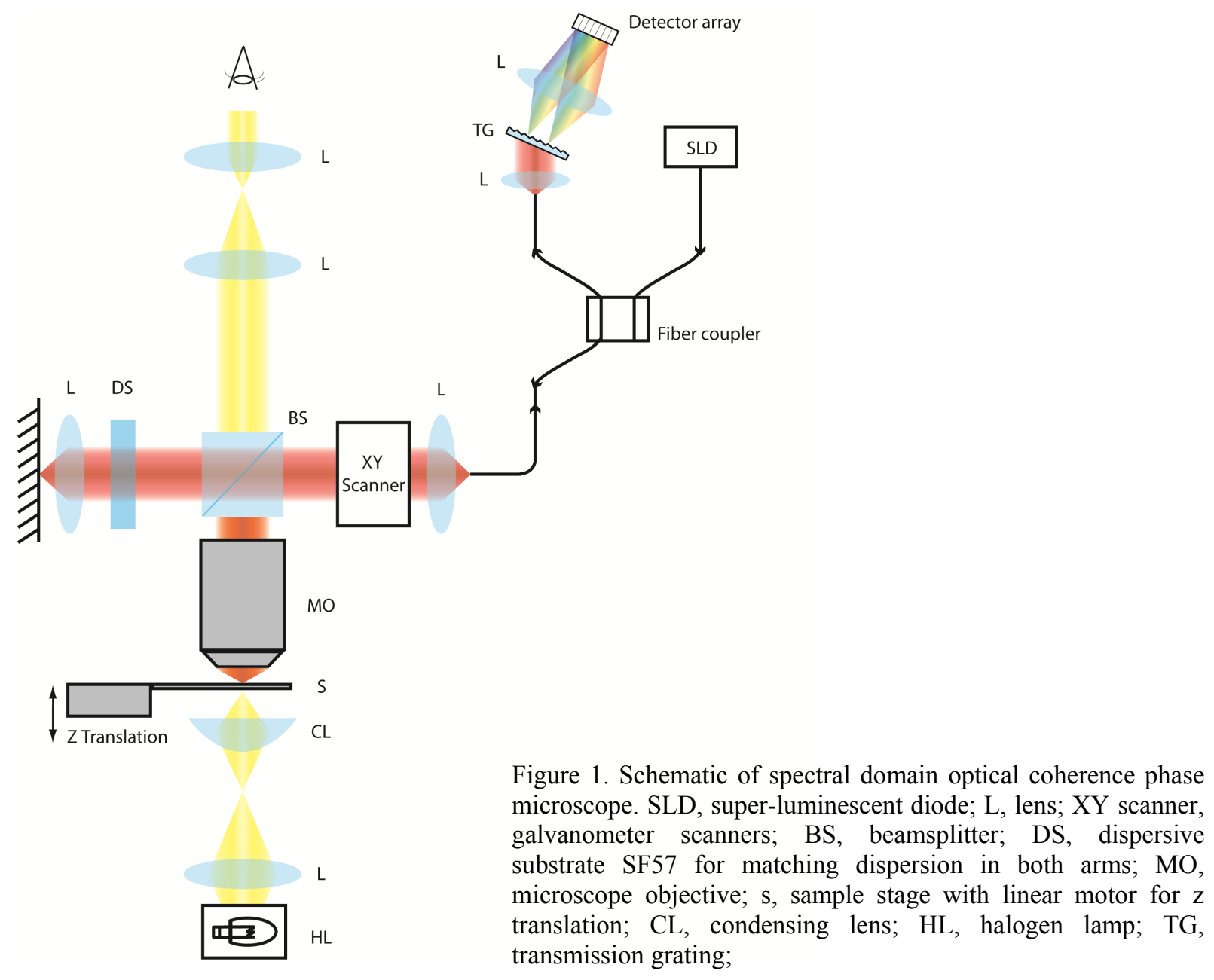

\subsection{Phase sensitivity}

The phase component of the fringe pattern is sensitive to the sub-wavelength changes in reflector position in depth and in a shot-noise limited optical setup, the theoretical limit of displacement sensitivity is determined by the signal to noise ratio of the measured fringed pattern ${ }^{3}$. Here we achieve high phase sensitivity by scanning through the Linnik interferometer setup, which provides two distinct advantages. First, the mechanical jitter introduced by X-Y scanners does not contribute to the noise floor of the system as it is common for both arms of the interferometer. The second advantage is that, Linnik interferometer setup allows for shot-noise regime detection of the fringe pattern as the reference arm intensity is controlled to be relatively higher than the sample reference arm, which is difficult with common path interferometer setups. 


\subsection{Transverse resolution and axial profiling}

The transverse resolution of OCPM setup is determined by the numerical aperture of the imaging optics. Here we have used a microscope objective with $0.8 \mathrm{NA}$ in sample arm, which provides a verified $1 \mu \mathrm{m}$ resolution in transverse plane. Figure 2 displays the obtained transverse with an air force target, group 7, where the parallel lines are 2.2 um apart. Three-dimensional profile of a sample can be obtained by unwrapping the phase component of fringe pattern in $\mathrm{X}-\mathrm{Y}$ plane. Figure 3 shows a color-coded three dimensional image of scanned air force target, group 7, where the color bar represents height of the metal deposit on glass surface.

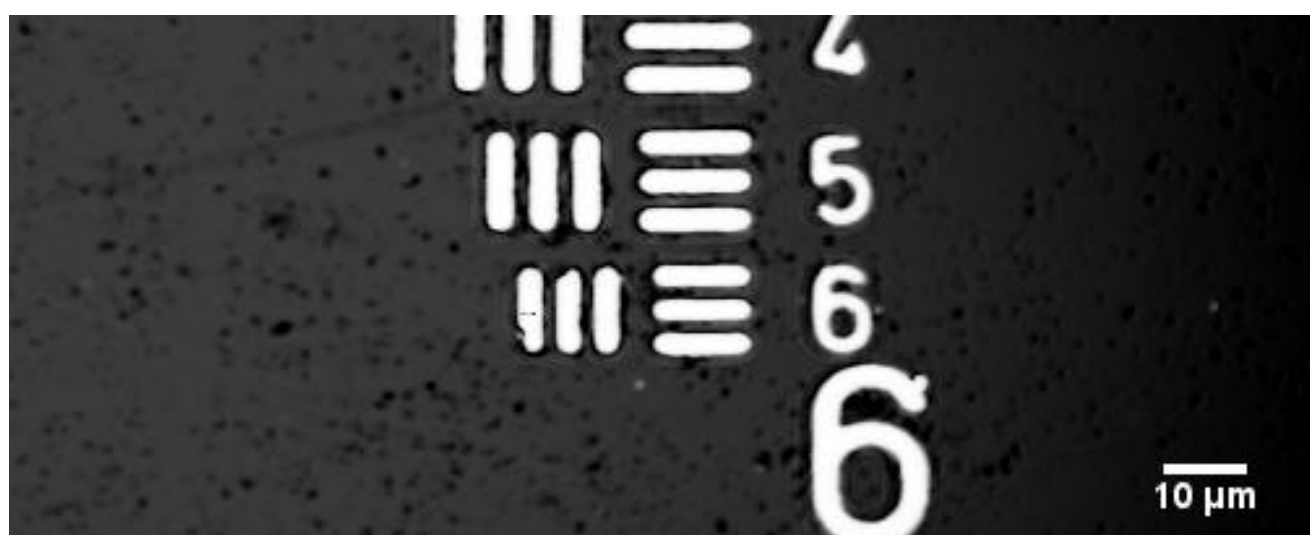

Figure 2. The transverse (X-Y) spatial resolution of the microscope is demonstrated with group 7 of Air Force Target, where parallel lines are $2.2 \mu \mathrm{m}$ apart.

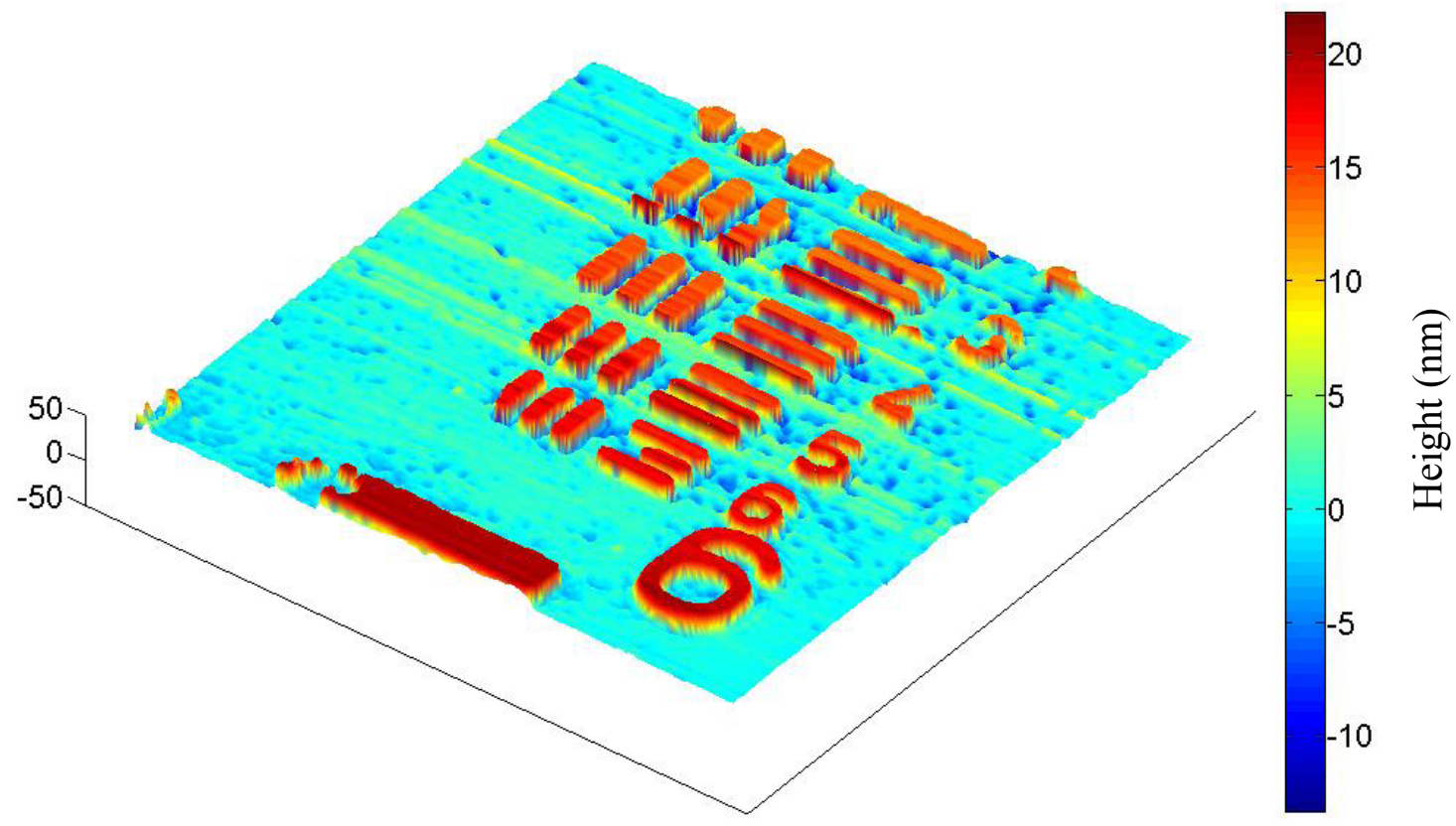

Figure 3. Color-coded three dimensional profile of Air Force Target group 7, which is obtained by two-dimensional unwrapping of the phase in $\mathrm{X}-\mathrm{Y}$ plane, color bar represents height in nanometer. 


\subsection{Preparation of cardiomyocytes}

For the differentiation of mouse embryonic stem (mES) cells into cardiomyocytes, 129s ES cells were cultured on feeder cells in DMEM medium supplemented with 20\% ES cell fetal bovine serum (FBS) until $80 \%$ confluent as previously described $^{5}$. Cells were trypsinised, resuspended and counted. A defined number of 400 cells/20 $\mu 1$ were used in hanging drops and cultured for 2 days until the embryoid bodies (EBs) were formed. Single EBs were plated separately into each well of a gelatin-coated $24-w e l l$ plate and cultured for $3+7$ days until the cardiomyocytes start beating spontaneously.

\subsection{Image acquisition}

Three dimensional structures of the cardiomyocytes and its phase contrast were obtained in three steps. First, stack of en-face images were obtained at $1 \mu \mathrm{m}$ steps in depth with active-low gated triggering, in order to avoid motion artifacts during image acquisition, because the movement of sample causes fringe pattern washout. The active-low triggering pattern was obtained by programming a clock to the frequency of cardiomyocyte beatings. In step two, area in the imaged volume was selected for high speed phase contrast scanning and then selected area was continuously scanned, each B-scan line at a time, at the rate of $217 \mathrm{k}$ Ascans/sec, for up to10 seconds to capture the displacement dynamics associated with contraction. In final step, the dynamic displacement images were obtained by integrating the phase of phase contrast data over time and registered with already acquired volumetric image.

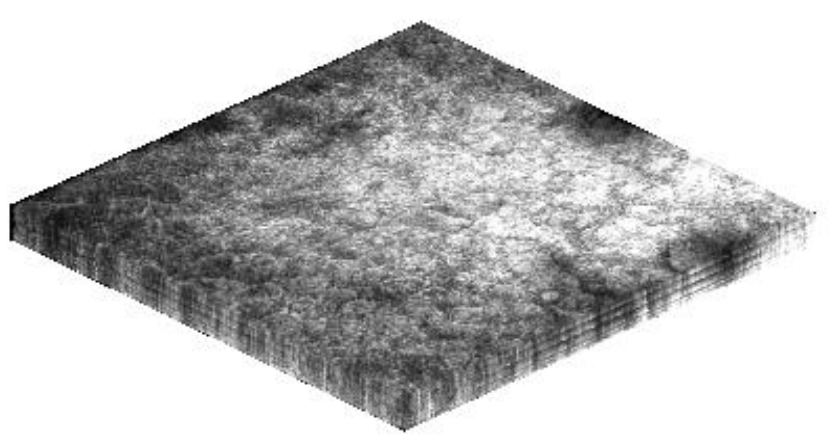

(a)

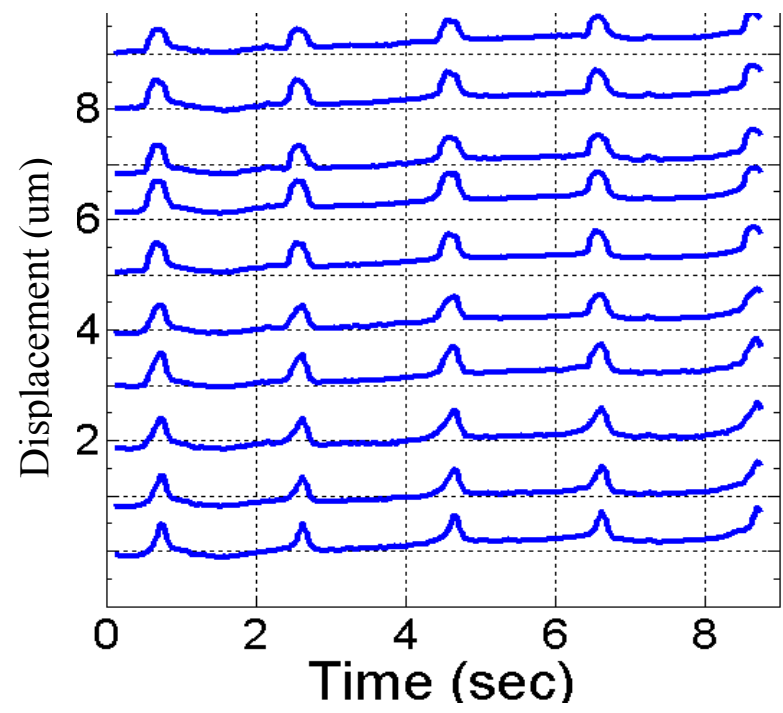

(c)

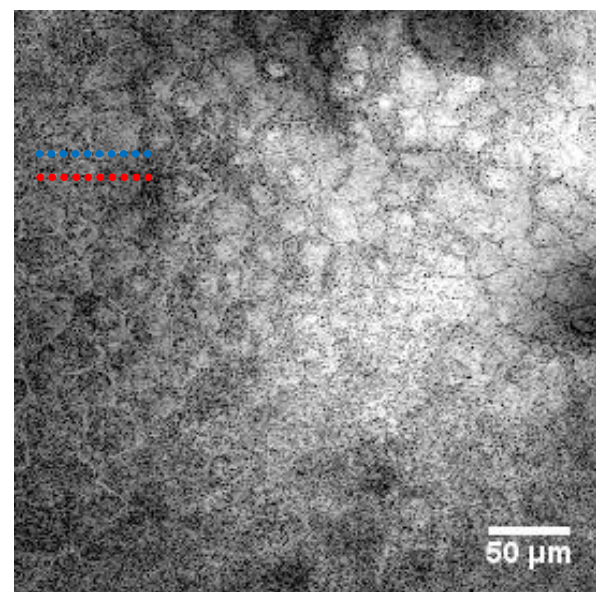

(b)

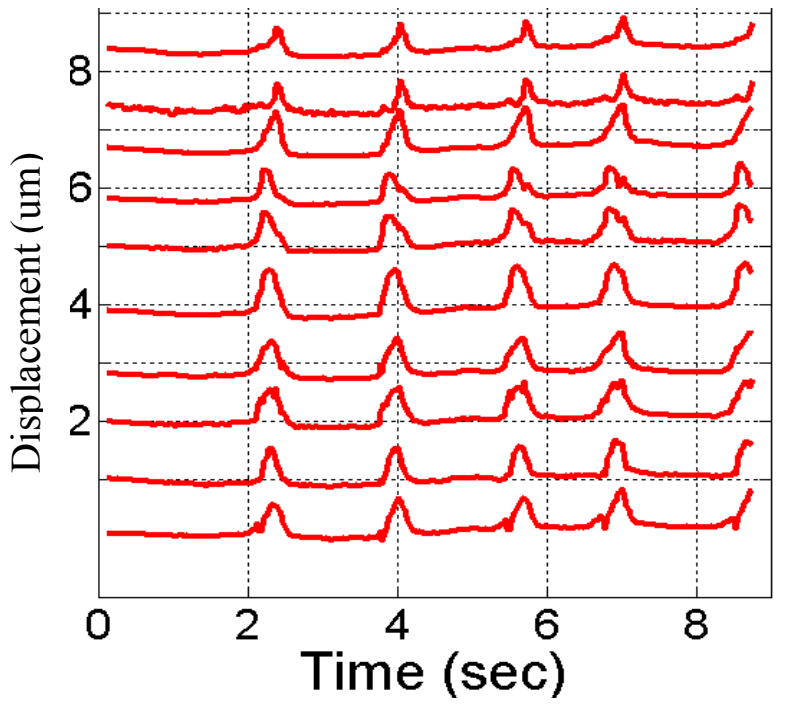

(d)

Figure 4. (a) Volumetric image of the cardiomyocyte obtained with 20 en-face optical sections in steps of $1 \mu \mathrm{m}$ in depth. 
4(b), one of the optical sections from the volume selected for high speed scanning for phase contrast. 4(c) \& 4(d) time series of axial displacement of sub-cellular structures in cardiomyocyte associated with contraction dynamics. The time series is obtained by integrating the phase contrast over time and y-axis is scaled to the axial displacement in micrometer.

\section{RESULTS}

First set of in-vitro experiments were performed on spontaneously active cardiomyocytes and the obtained volumetric image and one of its constituent optical sections is shown in figure $4 \mathrm{a} \& 4 \mathrm{~b}$ respectively. Figure $4 \mathrm{c}$ and $4 \mathrm{~d}$ are the time series of spatial locations marked by blue and red dots in figure $4 \mathrm{~b}$, arranged in the order left-right dots corresponding to top-bottom time-series. The time series are scaled to axial displacement in micrometers on y-axis and to time in seconds on x-axis. The quantitative measurement of the displacement provides information about the frequency of the cardiomyocyte beatings, spatio-temporally resolved to sub-cellular structures. Furthermore, strength of the cardiomyocyte beatings could be quantified as well, by identifying the cell types and bio-mass in the area of interest.

\section{CONCLUSION}

In conclusion, we quantitatively measured the sub-cellular structural displacements in cardiomyocytes associated with contraction dynamics and demonstrate that the OCPM can be used to study physiological dynamics at cellular level. Furthermore, this approach can be used to characterize the propagation and modulation of contraction dynamics in cardiomyocytes in relation to controlled variables, such as temperature, calcium concentration, etc., at cellular level and can facilitate basic research and drug discovery for various cardiomyopathy in-vitro models.

\section{REFERENCES}

[1] A.F. Fercher, C.K. Hitzenberger, G. Kamp, S.Y. El-Zaiat, "Measurement of intraocular distances by backscattering spectral interferometry," Opt. Commun. 117, 43 (1995).

[2] Michael Choma, Marinko Sarunic, Changhuei Yang, and Joseph Izatt, "Sensitivity advantage of swept source and Fourier domain optical coherence tomography," Opt. Express 11, 2183-2189 (2003).

[3] Choma M. A., Ellerbee A. K., Yang C., Creazzo T. L., and Izatt J. A., Spectral-domain phase microscopy. Opt. Lett. 30, 1162-1164 (2005).

[4] Joo C., Akkin T., Cense B., Park B. H., and de Boer J. F., Spectral-domain optical coherence phase microscopy for quantitative phase-contrast imaging. Opt. Lett. 30, 2131-2133 (2005).

[5] Victor A. Maltsev, Anna M. Wobus, Juirgen Rohwedel, Michael Bader, Jurgen Hescheler, "Cardiomyocytes differentiated in vitro from embryonic stem cells developmentally express cardiac-specific genes and ionic currents," Circulation Research. 75, 233-244 (1994) 